\title{
Experimente zum chemischen Anzünder mittelalterlicher Kriegsfeuer auf Kalziumoxidbasis
}

\author{
von Ferdinand Nibler*
}

In vielen Schriften, die von der Antike bis in die Neuzeit überliefert wurden, ist vom Gebrauch des Feuers zu kriegerischen Zwecken die Rede. Häufig sind dabei Rezepte für die Anfertigung von Brandsätzen zu finden, die oftmals auch einen Anzünder enthalten. Dieser chemische Anzünder auf Kalziumoxidbasis, der Kern der so genannten „automatischen Feuer“ (im Sinne von „sich selbst entzündend"), soll hier experimentell untersucht werden, um so zu einer besseren Beurteilung der überlieferten Rezepte zu kommen: Waren sie machbar? Waren sie wirksam? Sind die überlieferten Wirkungen realistisch?

Chemische Anzünder auf Kalziumoxidbasis sind so genannte ,automatische Feuer", welche sich paradoxerweise bei Wasserkontakt entzünden, weil der gebrannte Kalk $(\mathrm{CaO})$ beim Ablöschen starke Hitze entwickelt und geeignete Substanzen mit niedrigem Flammpunkt wie z.B. Schwefel zur Entzündung bringen kann. In Band 72 dieser Zeitschrift hat Wilfried Tittmann die Frage, ob es ,automatische“ Kriegsfeuer im Mittelalter gegeben habe oder hätte geben können, auf quellenkundlich-historischer Basis sowie im Kontext der aktuellen Salpeterfrage ausführlich diskutiert. ${ }^{1}$ Im selben Band hat der Verfasser dieser Miszelle dazu ergänzende Überlegungen auf naturwissenschaftlicher Basis angestellt und ist insbesondere dem physikalischchemischen Teilthema des chemischen Anzünders auf Kalziumoxidbasis als dem Herzstück der überlieferten Kriegsfeuer nachgegangen. ${ }^{2}$ Mangels experimenteller Erprobung musste allerdings die Frage noch offen bleiben, ob und inwieweit die tradierten Rezepte praxistauglich waren - starke Zweifel waren nämlich in mehrfacher Hinsicht angebracht. Verfasser hat es sich deshalb zur Aufgabe gemacht, das Problem nach Möglichkeit auf experimenteller Grundlage zu lösen und dazu einige grundlegende Versuche anzustellen.

Zur Illustration des aus den genannten Veröffentlichungen hinreichend bekannten Themas ${ }^{3}$ sollen hier stellvertretend für viele nur drei typische Re-

* Der Verfasser bedankt sich ganz herzlich bei Herrn Dr. Jürgen Knobloch, der die Untersuchungen im Laboratorium der Fa. Nitrochemie Aschau möglich gemacht hat, und bei Herrn Wilfried Tittmann, der als unermüdlicher Ratgeber und Diskussionspartner diese Arbeiten intensiv gefördert hat.

1 Wilfried Tittmann, Gab es selbstentzündliche Kriegsfeuer im Mittelalter? Anmerkungen zur Kalziumsalpeter-Theorie, in: Technikgeschichte 72, 2005, S. 275-302.

2 Ferdinand Nibler, Überlegungen zum chemischen Anzünder auf Kalziumoxidbasis, in: Technikgeschichte 72, 2005, S. 303-311.

3 Vgl. ebd., S. 304, wo auch weitere Erläuterungen zur Einteilung der Rezepte zu finden sind. 
zepte für selbstentzündliche Kriegsfeuer zitiert werden, die später auch ausgewertet werden müssen. Typisch sind diese drei Rezepte deshalb zu nennen, weil sie die drei möglichen Materialkombinationen repräsentieren und drei Entwicklungsstufen entsprechen: Der „Urzünder“ besteht nur aus Branntkalk und Schwefel (Typ 1), während weiterentwickelte Anzünder zusätzlich Übertragungsmassen in Form von Ölen oder Fetten besitzen, die als Brandbeschleuniger wirken und das Feuer auf schwer entzündliche Materialien übertragen können (Typ 2). Schließlich wurde laut spätmittelalterlichen Rezepten auch Salpeter als Sauerstoffträger hinzugemengt, wodurch rein theoretisch das heftigste Schadfeuer entstehen sollte (Typ 3).

Das nachfolgend erste und dritte Rezept sind aus dem anonymen Feuerwerkbuch (Sigle: FWB) entnommen, das Anfang des 15. Jahrhunderts entstanden sein dürfte, das zweite stammt aus dem Buch der probierten Künste von $1535:^{4}$

\section{(FWB 2295) Wie man macht das sich wasser anzündt}

Wiltu ein matery machen das sich wasser anzind /so nimm vngeleschtenn kalck vnd als vil schwebels an der wag /vnnd auß der matery mach ein dacht ${ }^{6} /$ vnd spreng darauff wasser so entzünt es sych /vnd geüst du öl darauff so erlischet es.

\section{(Helm 213/1 ${ }^{\top}$ ) Ein annders}

Item Nim venedigen ${ }^{8}$ kalck, gummi arabicum, schweffel, Lein öl temperier es durch einander, das ein matheri daruß werde, wie ein Confect mach darauß was du wilt, besprenge es mit wasser, oder Laß darauff Regnen, so zundt es sich selbs ane, vnnd brennt fewers Loß.

4 Zitate aus dem anonymen Feuerwerkbuch sind dem vom Verfasser erarbeiteten Papier „Das Feuerwerkbuch in synoptischer Darstellung zweier anonymer Originaltexte - Transkription und Textvergleich des Manuskriptes Ms 362 von 1432 und des so genannten Feuerwerkbuches von 1420 (gedruckt 1529) mit Anmerkungen zu den Texten“" entnommen. Diese mit vielen Anmerkungen und einer neuhochdeutschen Übertragung versehene Darstellung kann vom Verfasser über www.feuerwerkbuch.de bezogen werden. Rainer Leng, Franz Helm und sein „Buch von den probierten Künsten“. Ein handschriftlich verbreitetes Büchsenmeisterbuch in der Zeit des frühen Buchdrucks (Imagines medii aevi. Interdisziplinäre Beiträge zur Mittelalterforschung, Bd. 9), Wiesbaden 2001; Rezension dazu von W. Tittmann in: Technikgeschichte 71, 2004, S. 171-173. Die nachfolgende Nummerierung der Rezepte folgt der jeweiligen Edition bei Nibler bzw. Leng.

5 Nibler (wie Anm. 2), S. 304.

6 „dacht" = „daucht“ => Docht. Vgl. J.A. Schmeller, Bayerisches Wörterbuch, 2 Bde. in 4 Teilen, 1827-37, 2. von G.K. Frommann bearbeitete Ausgabe, München 1872-77, Reprint München 1985, Bd. I/1, Sp. 484.

7 Leng (wie Anm. 4), S. 213.

8 „venedigen kalck“ ist nicht erklärbar. Könnte es eine Verstümmelung von „lebendigem kalck" sein? Lebendiger Kalk ist ungelöschter Kalk.

9 Nibler (wie Anm. 2), S. 304. 
(FWB 203 ${ }^{9}$ ) Wie man gar ain wunderlich puluer machen sol dasselb puluer ouch die art haut wa man es hin legt vnd es trucken ligt so ennbrint es von im selb nit aber wenne es darvff regnot oder das es besch [ut $\left.{ }^{10}\right]$ wirt das es naß geworden were so ennbrint es dauon

Wilt du ain sölich puluer machen dauon die rubric sagt so nym salniter $\mathrm{z}^{11} \mathrm{v}$ sulpur $\mathrm{z} j$ carbones $\mathrm{z} ß$ vnd puluer das vnder ain annder vnd niem ainen wyssen kisling stain vnd brenne den $\mathrm{z} u$ ainem kalck vnd stoß den vngelesten kalck wol vnd $t \mathrm{u} z$ i des kalcks darz $u$ vnd dryer pfenning swer campffer vnd temperiere das wol vnder ain annder vnd lauß es wol trucken werden.

Das Rezept FWB 229 ist typisch für den chemischen Anzünder vom Typ 1 auf Kalziumoxidbasis, der kurz und treffend beschrieben wird: „nimm ungelöschten Kalk und ebensoviel Schwefel ... spreng darauf Wasser, so entzündt es sich.“ Im Rezept Helm 213/1 wird die Konsistenz der Mischung des Typs 2 mit Leinöl (oder einem anderen Öl, Fett Wachs usw.) genauer beschrieben durch den Hinweis ,das ein matheri daraus werde, wie ein Confect". Im Rezept FWB 203 des Typs 3 tritt ergänzend noch Salpeter hinzu. Dieses Rezept gibt Aufschluss über die Konsistenz der Anzünder ohne Ö1, Fett usw., denn es heißt ,um solch ein Pulver zu machen ... so pulvere (die Bestandteile) untereinander ... und stoße (in einem Mörser) den ungelöschten Kalk“". Danach ist die Anzündmasse entweder ein Pulver oder ein „Konfekt“.

Kernstück der chemischen Anzünder ist in allen Fällen die chemische Reaktion beim Löschen des Kalkes, die stark exotherm ist: ${ }^{12}$

$$
\mathrm{CaO}+\mathrm{H}_{2} \mathrm{O} \rightarrow \mathrm{Ca}(\mathrm{OH})_{2}+\mathrm{W}
$$

Diese Reaktion liegt allen ,,automatischen“ Feuern zugrunde. Die in der Reaktionsmasse freiwerdende Energie W=1,79 kWs $/ \mathrm{cm}^{3}$ soll den Schwefel bis zur Selbstentzündung bei $260^{\circ} \mathrm{C}$ erhitzen und damit das Feuer initiieren.

Einen Überblick über die wichtigsten überlieferten Rezepte findet der Leser in den schon genannten Aufsätzen von Tittmann und Nibler. Zur eingehenderen Information ist auf die dort angegebenen Quellen zu verweisen. Die Mehrzahl der überlieferten Texte stammt vom Anfang des 15. Jahrhunderts, doch sind einige der Rezepte auch älter, wie aus der ausführlichen Diskussion hervorgeht, die Romocki der Frühgeschichte dieser Brandsätze widmet. ${ }^{13}$ Wenn man Romocki folgt, sind die ersten Brandsätze auf CaOBasis zwischen dem 6. und 10. Jahrhundert in Byzanz entwickelt worden. ${ }^{14}$

10 Ergänzung nach den Codices DM/MM203: „,beschut wird“ = „,beschüttet wird“ => „Wasser reichlich zuführen“, WM203 hat ,... oder wenn man es begevst mit wasser ...“.

11 Auf die mögliche Lesart , $\mathrm{Z}^{\prime \prime}=$ Pfund hat dankenswerterweise Wilfried Tittmann, Bochum, hingewiesen, ebenso auf ,B“ $=$,semi“" $=$, ,halb“.

12 Für Details wird auf Nibler (wie Anm. 2), S. 305ff. verwiesen.

13 Siegfried Julius v. Romocki, Geschichte der Explosivstoffe, Hildesheim 1983 (Nachdruck der Ausgabe Berlin 1895), S. $9 \mathrm{ff}$.

14 Ebd., S. 13. 
Diese frühe Entstehung einiger Rezepturen wird nach Ansicht des Verfassers durch die Tatsache bestätigt, dass nur in ganz wenigen - nach Kenntnis des Verfassers nur zwei - Rezepturen Salpeter erscheint, der in Byzanz noch unbekannt war. Letztmals - nach Kenntnis des Verfassers - wurden Feuerrezepte dieser Art (d.h. Typ 1 bis 3) von Franz Helm in seinem Buch von den probierten Künsten 1535 zusammengestellt, das 1625 erstmals gedruckt wurde. ${ }^{15}$ D.h. die älteren Rezepte vom Typ 1 und 2 für selbstzündende Brandsätze wurden rund 1000 Jahre lang wieder und wieder abgeschrieben! Ob sie allerdings auch 1000 Jahre lang erfolgreich eingesetzt wurden, bezweifelt Verfasser aufgrund seiner theoretischen Überlegungen ${ }^{16}$ und der unten wiederzugebenden praktischen Erfahrungen ganz entschieden.

Als Fazit seiner Überlegungen hat Verfasser die Forderung nach einer experimentellen Überprüfung der Rezepte erhoben. Als praktische Voraussetzung für eine experimentelle Untersuchung müssen nun zunächst - möglichst! alle Rezepte gesichtet werden, um die Kenntnis der Zusammensetzungen zu erhalten. Diese im ersten Moment fast übergroß erscheinende Aufgabe schrumpft bei näherem Zusehen ganz beträchtlich, weil sehr viele Rezepte zeitlich und örtlich mehrfach überliefert wurden, wie Tittmann dargestellt hat. ${ }^{17}$ Es gibt also im Grunde genommen nur drei verschiedene Typen des chemischen Anzünders, wie sie oben bereits angeführt wurden:

- Typ 1: Kalziumoxid und Schwefel (z.B. Rezept FWB 229)

- Typ 2: Kalziumoxid, Schwefel und brennbare Substanz(en) ${ }^{18}$ (z.B. Rezept Helm 231/1)

- Typ 3: Kalziumoxid, Schwefel, brennbare Substanz(en) und Salpeter (z.B. Rezept FWB 203).

Typ 3 erscheint im ersten Moment wegen des Sauerstoffträgers Salpeter als die optimale Lösung für das Entzünden eines starken Feuers, aber der ,nasse Anzünder", der durch Löschen des Kalkes entsteht, würde den Salpeter unvermeidbar ebenfalls ,nass“ und damit als Sauerstoffträger wohl unbrauchbar machen. Die nur in zwei Einzelfällen überlieferte Mischung vom Typ 3 wurde darum aus der weiteren Untersuchung herausgelassen. Trotzdem wird ein solches salpeterhaltiges Rezept aus dem Feuerwerkbuch angeführt zur Information und weil es wegen der Angaben über die Zubereitung der Mischung und die Mengen der Zutaten noch auszuwerten ist.

Für alle drei Typen von Anzündern sind unterschiedliche Mengenverhältnisse in der Zusammensetzung möglich und wohl auch zu erwarten. Nur wenige der überlieferten Rezepte enthalten indes absolute oder wenig-

15 Leng (wie Anm. 4), S. 151.

16 Nibler (wie Anm. 2), S. $305 \mathrm{ff}$.

17 Tittmann (wie Anm. 1), S. 295ff.

18 Hierunter fallen Substanzen wie Wachs, Pech, Petroleum usw., die als „Übertragungsladung“ wirkend ein Sekundärfeuer aus dem Primärfeuer des Anzünders vom Typ 2 oder auch 3 entfachen und unterhalten können. 
stens relative Angaben über die Zusammensetzung - diese wenigen Rezepte müssen also ausgewertet werden, um historisch haltbare Experimente zu ermöglichen. Die schematische Übersicht zeigt das Ergebnis:

Auswahl von vier Rezepten mit absoluten bzw. relativen Mengenangaben: (Umrechnung der Komponenten auf jeweils $100 \mathrm{~g} \mathrm{CaO}$ entsprechend $\approx 3$ Unzen)

Aus dem Bellifortis (1405): ${ }^{19}$

Blatt $91 b$

Kerze, die im Wasser angezündet wird und brennt

$\rightarrow \quad 1 / 2$ Pfd. ${ }^{20}$ Wachs, 2 Unzen ${ }^{21}$ Schwefel, 2 Unzen Kalk, 1/2 Unze Petroleum

$\rightarrow \quad 250 \mathrm{~g}$ Wachs, $60 \mathrm{~g} \mathrm{~S}, 60 \mathrm{~g} \mathrm{CaO}, 15 \mathrm{~g}$ Petroleum

$\rightarrow 100 \mathrm{~g} \mathrm{CaO}, 100 \mathrm{~g} \mathrm{~S}, 420 \mathrm{~g}$ Wachs, $25 \mathrm{~g}$ Petroleum $+33 \mathrm{ml} \mathrm{H}_{2} \mathrm{O}$

Blatt $101 b$

Ein sehr gutes Feuer:

$\rightarrow \quad 2$ Tle. Schwefel, 3 Tle. Kalk, 6 Tle. Wachs, etwas Petroleum

Blatt $102 a$

$\rightarrow 100 \mathrm{~g} \mathrm{CaO}, 66 \mathrm{~g} \mathrm{~S}, 200 \mathrm{~g}$ Wachs, $\approx 10 \mathrm{~g}$ Petroleum $+33 \mathrm{ml} \mathrm{H}_{2} \mathrm{O}$

Damit durch Regen ein Haus angezündet werde:

$\rightarrow 2$ Unzen Schwefel, 3 Unzen Kalk, $\approx 1$ Unze Petroleum

$\rightarrow \quad 60 \mathrm{~g} \mathrm{~S}, 90 \mathrm{~g} \mathrm{CaO}, 30 \mathrm{~g}$ Petroleum

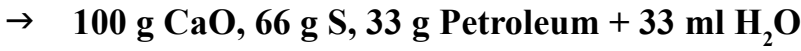

\section{Aus dem Feuerwerkbuch von 1420:}

FWB 229

Wie man macht das sich wasser anzündt.

$\rightarrow \quad 1$ Tl. Kalk, 1 Tl. Schwefel

$\rightarrow 100 \mathrm{~g} \mathrm{CaO}, 100 \mathrm{~g} \mathrm{~S}+33 \mathrm{ml} \mathrm{H}_{2} \mathrm{O}$

Die Anzündmassen sind durchweg als Mischung von gepulvertem $\mathrm{CaO}$ und $S$ anzusehen gemäß der Anweisung: ,vnd puluer das vnder ain annder“ im oben stehendem Rezept FWB 203. Sind Wachs oder andere Beimengungen einzuarbeiten, so muss eine „,konfektartige Masse“ durch Kneten oder durch Stoßen in einem Mörser gemacht werden. Auch diese Anweisung spricht für die innige Mischung der Materialien. Dieser Aspekt ist für die Bewertung der Rezepte und ihrer Tauglichkeit mitentscheidend!

Bei den wenigen etwas genaueren Mengenangaben in den vier auswertbaren Rezepten erstaunt die absolut geringe Menge an verwendetem Material: zwei oder drei Unzen Kalk bzw. Schwefel entsprechen gerade etwa je 100 g,

19 Konrad Kyeser, Bellifortis, Bd. 1: Faksimile der Göttinger Pergament-Handschrift, Bd.

2: Umschrift und Übersetzung von Götz Quarg, Düsseldorf 1967.

201 Pfund $\approx 500 \mathrm{~g}$.

211 Unze $\approx 30 \mathrm{~g}$. 
d.h. die gesamte Reaktionsmasse liegt danach nur bei etwa 200 g. Es ist kaum vorstellbar, dass damit ein echtes Schadfeuer zu militärischen Zwecken entzündet werden konnte.

Trotz dieser Bedenken wurden die nun zu schildernden Versuche, wenn nicht anders angegeben, zunächst immer mit der Grundmenge $100 \mathrm{~g} \mathrm{CaO}+$ $100 \mathrm{~g} \mathrm{~S}$ angesetzt.

\section{Versuche mit Rezeptur Typ 1, intensiv gemischt}

Beim ersten Versuch wurden $\mathrm{CaO}$, das nur gepulvert zur Verfügung stand, und der ebenfalls gepulverte Schwefel sorgfältig in einem $20 \mathrm{~cm}$-Uhrglas gemischt. Es wurde die stöchiometrisch korrekte Wassermenge von $33 \mathrm{ml}$ zugesetzt, aber der Schwefel entzündete sich nicht, weil die erreichte Temperatur nicht hoch genug war (gemessen wurden nur $200^{\circ} \mathrm{C}$ ). Der Grund dafür war, dass im Verlauf der sich über ca. 60 bis 90 Sekunden hinziehenden Reaktion das Wasser, das noch nicht beteiligt, aber bereits $100^{\circ} \mathrm{C}$ heiß war, als Dampf aus der Reaktion ausschied. Der Versuch wurde daher mit mehr als der eigentlich korrekten Wassermenge mehrfach wiederholt, bis es bei ca. $100 \mathrm{ml}$ Wasser, also der dreifachen Soll-Menge, endlich im Kern der Reaktionsmasse zu der erwarteten Temperatur von $>300^{\circ} \mathrm{C}$ kam als Voraussetzung für die Selbstentzündung des Schwefels. Das Ergebnis war enttäuschend, weil sich zeigte, dass das Wasser tatsächlich mengenmäßig sorgfältig dosiert in einem einzigen zügigen Guss zugeführt werden musste mit einem geschätzten zulässigen Fehler von $\leq 10 \%$ ! Ein Aktivieren des Anzünders durch Regen oder durch einfaches Besprengen oder Beschütten mit Wasser, wie in den Rezepten angegeben, ist demnach nicht möglich, es sei denn, die Regenmenge „stimmt zufällig“.

Bei mehreren weiteren Versuchen waren die Materialien gut gemischt, aber es kam trotz der erhöhten Wasserzugabe wieder nicht zur Entzündung des Schwefels, der zwar die kritische Temperatur $>260^{\circ} \mathrm{C}$ erreichte, aber vom Luftsauerstoff ,abgeschirmt“" war.

\section{Versuche mit Rezeptur Typ 1, ungemischt}

Bei Wiederholungen wurde darum - gegen die ausdrückliche Anweisung in den Rezepten ${ }^{22}$ - auf die sorgfältige Mischung verzichtet, so dass unvermengte „Kalkbereiche“ und „Schwefelbereiche“ mit Oberflächen von $\approx 3$ bis $\approx 5 \mathrm{~cm}^{2}$ in dem Versuchsgefäß vorhanden waren. Jetzt kam es zur Entzündung des Schwefels, wie Abb. 1 zeigt. ${ }^{23}$

22 Trotz der festen Absicht, keinen funktionsfähigen Anzünder zu entwickeln, sondern nur die Originalrezepte zur Probe nachzumachen, erschienen diese und später noch weitere kleinere Abweichungen von der strengen Forderung der Rezepte vertretbar, um wenigstens ein paar Teilergebnisse zu erhalten.

23 Die farbigen Abbildungen zu den Versuchen sind im Internet abrufbar unter: http:// www.edition-sigma.de/TG/H1_07/AbbNibler.PDF. 


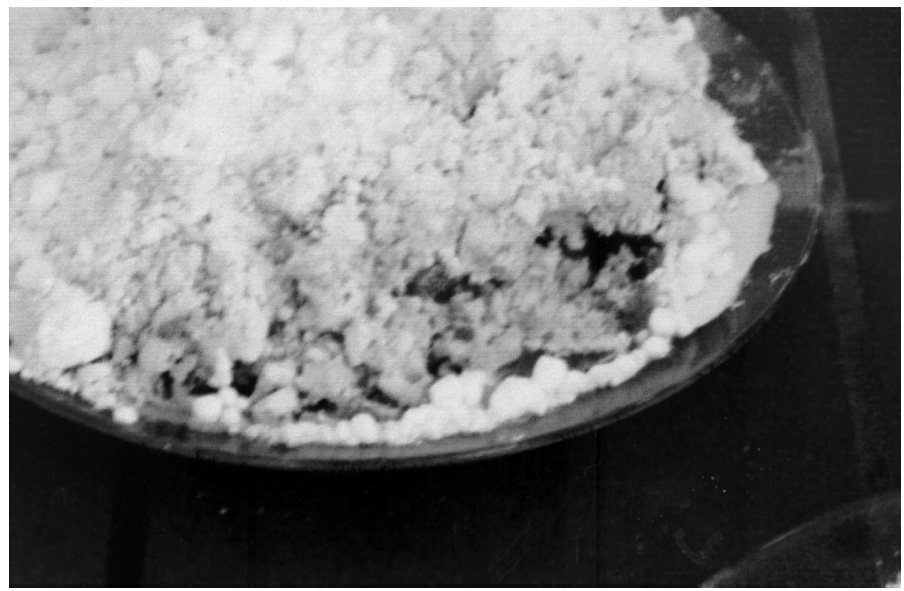

Abb. 1: Der Schwefel hat sich entzündet - aber er brennt nur mit einer kleinen blauen Flamme, Flammlänge etwa $5 \mathrm{~mm}$, in einer langsam fortschreitenden Front (hier: der dunkelgraue Fleck am rechten unteren Rand - die farbige Abbildung ist im Internet abrufbar unter: http://www. edition-sigma.de/TG/H1_07/AbbNibler.PDF). Die hier abgebildete Situation, etwa fünf Minuten nach der Reaktion, kann sich so bis zu einer Stunde hinziehen!

Dass sich kein kräftiges Feuer ergab, war zwar enttäuschend, aber erklärlich: Schwefel schmilzt bei $119^{\circ} \mathrm{C}$, die Schmelze ist hellgelb und dünnflüssig. Bei höherer Temperatur aber wird die Schmelze dunkelbraun - das ist in Abb. 1 rechts vom Feuer deutlich erkennbar - und zäh wie Pech, d.h. die Fließfähigkeit hat bei ca. $200^{\circ} \mathrm{C}$ ein ausgeprägtes Minimum. Dementsprechend kann der Schwefel nicht nach unten ablaufen, sich nicht ansammeln und keine größeren Feuerherde bilden. Die besten Ergebnisse wurden erzielt, wenn der Schwefel nur auf den Kalk aufgeschüttet wurde, wie Abb. 2 zeigt. Insgesamt lag bei einer so zubereiteten Kalk-Schwefel-Mischung oder besser -Kombination die Entzündungsrate bei ca. 60 bis $70 \%$.

Abb. 2: Schwefel, der auf den Kalk geschüttet war und erst wegen der Volumenvergrößerung des reagierenden Kalkes seitlich abgerutscht ist, hat sich entzündet. Die Temperatur im aktiven Kern ist deutlich über $300^{\circ} \mathrm{C}$. Der Durchmesser der Glasschale ist $20 \mathrm{~cm}$, ca. $150 \mathrm{~g}$ Kalk und ca. $100 \mathrm{~g}$ Schwefel wurden verwendet (farbige Abbildung im Internet: http://www.edition-sigma. de/TG/H1_07/AbbNibler.PDF).

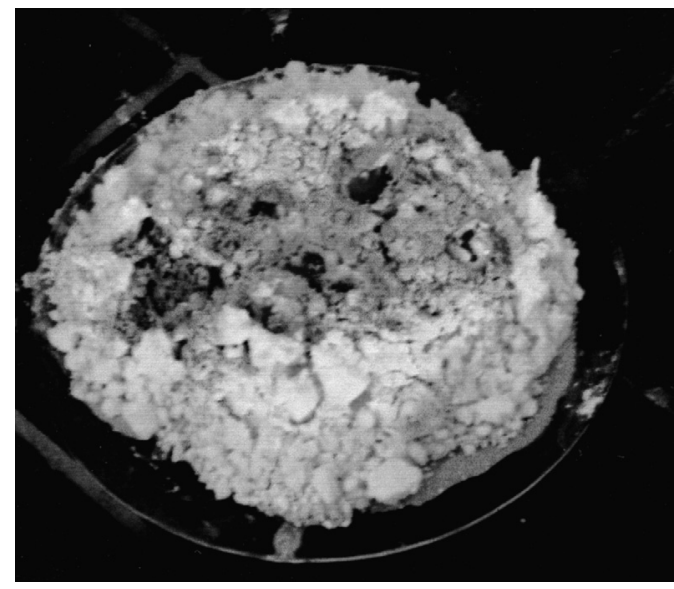




\section{Versuche mit Rezeptur Typ 2, intensiv gemischt}

Diese relativ hohe Erfolgsrate darf aber nicht darüber hinwegtäuschen, dass mit Massen im 100 g-Bereich - wie hier noch verwendet - kein echtes Schadfeuer entzündet werden kann - die kleinen Schwefelflämmchen sind viel zu schwach dazu und würden bei Regen wohl nahezu sofort erlöschen! Aber das „primäre Feuerchen“ kann unter günstigen Umständen zur Entzündung eines „kräftigen Sekundärfeuers“ dienen, wenn man Mischungen von Typ 2 mit Wachs oder Pech oder Petroleum ${ }^{24}$ als Brandmasse für das Sekundärfeuer ansetzt. Wie schon aufgrund der früheren Vorüberlegungen befürchtet, ${ }^{25}$ ist die Beimischung von Petroleum nicht unkritisch. Bei den ersten Versuchen wurde - wie bei den anfänglichen Versuchen mit Typ 1 - aus Petroleum, Kalk und Schwefel ein „matheri ... wie ein Confect “ gemacht, d.h. die drei Materialien wurden gut gemischt. Dass es dabei zu keiner Entzündung des Schwefels kam und natürlich erst recht nicht zu einer Entzündung des Petroleums, erklärt sich durch die zusätzliche ,abschirmende Wirkung“ des Petroleums: Dadurch kam es zu einer Verlangsamung der Kalk-Wasser-Reaktion (als Folge davon ergab sich eine niedrigere Maximaltemperatur von nur etwa $200^{\circ} \mathrm{C}$ ) und der Luftsauerstoff war vom Schwefel noch besser ferngehalten worden! Die Abb. 3 zeigt das negative Ergebnis.

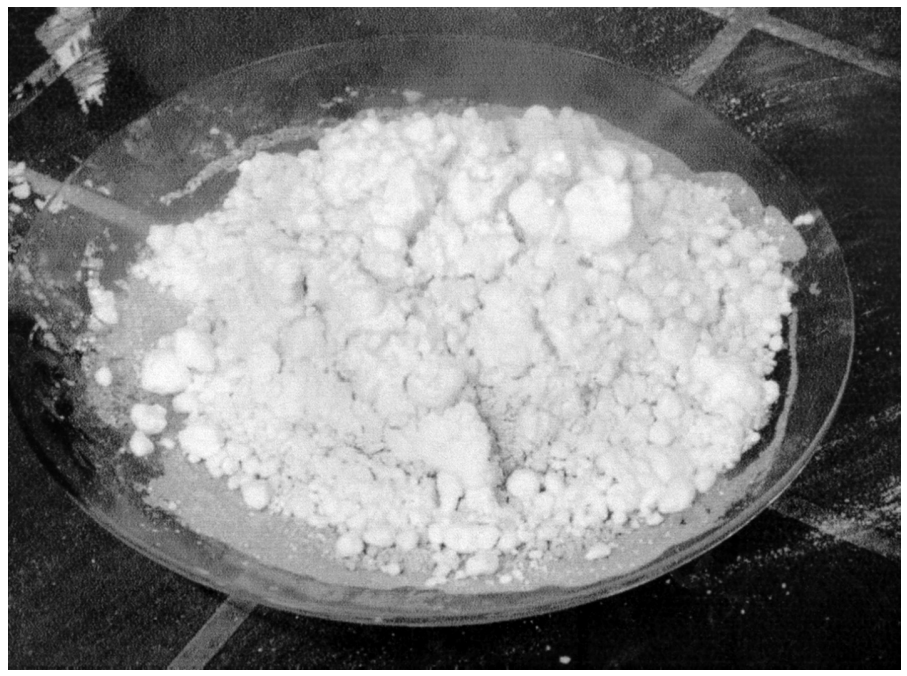

Abb. 3: Eine Mischung aus Kalk, Schwefel und Petroleum reagiert auf die Wasserzugabe weniger heftig und langsamer als eine reine Kalk-Schwefel-Mischung. Dementsprechend kommt es nicht zur Entzündung des Schwefels und erst recht nicht zur Entzündung des Petroleums (farbige Abbildung im Internet: http://www. edition-sigma.de/TG/H1_07/Abb Nibler.PDF).

24 Hier, bei dieser Versuchsreihe, wurde nur handelsübliches Petroleum verwendet.

25 Nibler (wie Anm. 2), S. 309. 


\section{Versuche mit Rezeptur Typ 2, ungemischt}

Deshalb wurde eine kleine Variation der Versuchsanordnung erprobt: Der mit Petroleum vermischte Schwefel wurde in ein „Nest“ aus Kalk gebettet, um das außen dazu geschüttete Wasser und das Petroleum-Schwefel-Gemisch innen zu trennen und wenigstens die Grundreaktion nicht durch das Petroleum zu hemmen. Die Grundreaktion lief auch programmgemäß ab, aber die steigende Temperatur führte dazu, dass sich der Schwefel im Petroleum löste und diese dünnflüssige Lösung im Kalk „versickerte“. Erst bei der Entleerung der Glasschale wurde die nach Abkühlung wieder erstarrte Schmelze oder Lösung an der tiefsten Stelle vorgefunden. Natürlich kam es weder zu einem „Primärfeuer“ noch zu einem „Sekundärfeuer“, die „Abschirmung“ war und blieb wirksam! Die Abb. 4 zeigt diese Versuchsanordnung als einen von mehreren vergeblichen Versuchen, ein Sekundärfeuer durch Anzünden des Petroleums zu erhalten. Obwohl im Inneren der Reaktionsmasse Temperaturen bis zu $335^{\circ} \mathrm{C}$ (an der oberen Messgrenze des Thermometers!) in guter Übereinstimmung mit den abgeschätzten möglichen $\approx 400^{\circ} \mathrm{C}$ gemessen wurden, entstand der Eindruck, dass die zu geringe Oberflächentemperatur der Grund für das „zu kleine Schwefelfeuer“ hätte sein können, ebenso für das Nichtentzünden des Petroleums. Das deckt sich auch mit einem Ergebnis der früheren Überlegungen, ${ }^{26}$ wonach die Größe des aktiven Volumens eine entscheidende Rolle spielt, d.h. die gewünschte Anzündwirkung wird mit einer

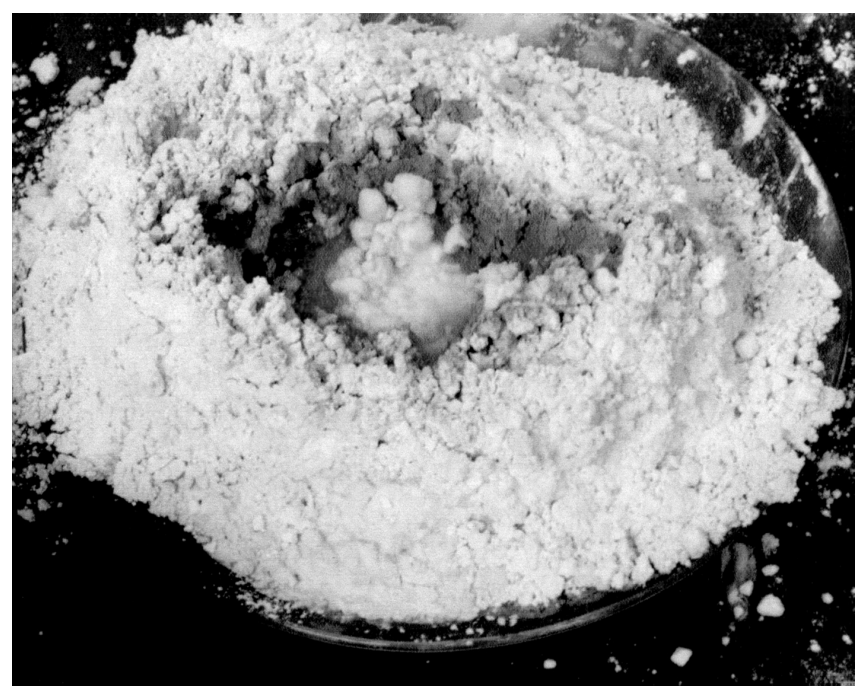

Abb. 4: Schwefel-Petroleum-Mischung im Kalk-Nest, einer der verschiedenen Versuche, mit denen ein wirksames Sekundärfeuer erzeugt werden sollte. Der Schwefel hat sich zum größten Teil gelöst, die dünnflüssige Lösung ist im Kalk versickert (farbige Abbildung im Internet: http://www.edition-sigma.de/TG/H1_07/AbbNibler.PDF). 
größeren aktiven Masse leichter erreicht als mit einer zu kleinen. Dabei dürfte die Gesamtenergie der Reaktion und die Oberfläche des aktiven Volumens eine entscheidende Rolle spielen.

\section{Versuche mit Rezeptur Typ 2, größere Reaktionsmasse}

Die Versuchsanordnung wurde völlig verändert: Die CaO-Masse wurde von $100 \mathrm{~g}$ auf ca. 2,5 kg erhöht, die Schwefel-Masse wurde entsprechend auf ca. $1 \mathrm{~kg}$ erhöht.

Das $\mathrm{CaO}$ wurde ca. $3 \mathrm{~cm}$ hoch in einer Blechwanne glatt gestrichen, so, dass sich wegen der abfallenden Ränder eine Fläche von ca. $25 \mathrm{~cm}$ x $15 \mathrm{~cm}$ ergab, über die der Schwefel als knapp $3 \mathrm{~cm}$ dicke Schicht verteilt wurde. Im Randbereich des Schwefels wurden an einer Stelle mit $\approx 10 \mathrm{~cm}$ Randlänge $\approx 20$ $\mathrm{ml}$ Petroleum eingebracht, ebenso im Mittelbereich auf einer Fläche von $\approx 10 \mathrm{~cm}^{2}$. Die deutlich größeren Massen der reagierenden Materialien entsprechen in etwa den in den Rezepten FWB 203 (s.o.) und FWB 231 angegebenen Massen. ${ }^{27}$ Leider sind aber diese Rezepte in sich nicht schlüssig, weil das erste

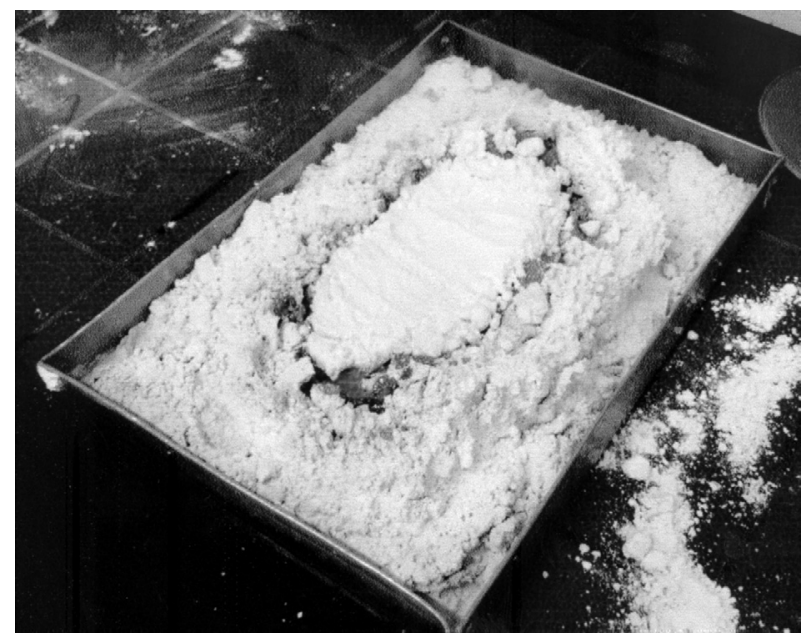

Abb. 5: Der „Großversuch“ mit $\approx 2,5 \mathrm{~kg} \mathrm{Kalk,} \approx 1 \mathrm{~kg}$ Schwefel, $\approx 40 \mathrm{ml}$ Petroleum knapp zwei Minuten nach Beginn der Reaktion. Die dunkelbraune Schwefelschmelze deutet auf eine Oberflächentemperatur von etwa 200 bis $250^{\circ} \mathrm{C}$, also noch unter der Entzündungstemperatur von $260^{\circ} \mathrm{C}$. Unmittelbar nach dieser Aufnahme, gut zwei Minuten nach Reaktionsbeginn, begann der Schwefel zu brennen (farbige Abbildung im Internet: http://www.edition-sigma.de/ TG/H1_07/AbbNibler.PDF).

27 Vgl. Tittmann (wie Anm. 1), S. 297f., Rezept FWB 203 aus dem Münchener Clm 30150 entnommen. Es handelt sich um eine späte Variante des so genannten Agarener-Rezeptes, das um 1300 nur 5 ,Teile“, 1405 sogar nur 1/2 Pfund Branntkalk angibt und erst im FWB auf ,5 Pfund lebendigen Kalk“ kommt; vgl. ebd., S. 286, 289 (Nr. 4) u. 298. Die aus dem Rahmen fallende große Reaktionmasse des Agarener-Rezeptes hängt vermutlich damit zusammen, dass Alexander der Große damit wahlweise die Häuser, Städte oder das Land der Araber (Agarener) verbrannt haben soll. 
Salpeter enthält, das zweite keinen Schwefel, so dass aus ihnen lediglich die Mengenangaben ,einige Pfund“ übernommen werden können.

Im Randbereich der Wanne wurde nun 11 Wasser eingefüllt. Die sofort beginnende Reaktion des $\mathrm{CaO}$ mit Wasser erhitzte die Reaktionsmasse so, dass nach ca. einer Minute der Schwefel am Rande bei ca. $120^{\circ} \mathrm{C}$ zu schmelzen begann. Die Veränderung der Farbe des geschmolzenen Schwefels zu Dunkelbraun ließ auf die Temperatur an der Schwefeloberfläche schließen, nämlich auf 200 bis $250^{\circ} \mathrm{C}$. Dieser dunkelbraune geschmolzene Schwefel ist zäh wie Pech! Deshalb kann er nicht fließen und deshalb kann sich das Feuer auch nicht ausbreiten! Die Abb. 5 zeigt die Versuchsanordnung in diesem Zustand ca. zwei Minuten nach Reaktionsbeginn.

Unmittelbar danach, also nach gut zwei Minuten, kam es zur Selbstentzündung des Schwefels, der von da an mit der üblichen kleinen blauen Flamme brannte, die auch schon an den anderen Versuchen beobachtet wurde. Die entscheidende Änderung trat ca. drei Minuten nach Reaktionsbeginn ein: Das Petroleum in der Mitte der Schwefelplatte entzündete sich, so dass ab jetzt ein hell loderndes Feuer brannte, das jederzeit auf anderes brennbares Material hätte übergreifen können. Nach ca. zehn Minuten begann auch das Petroleum am Rande zu brennen, so dass ab diesem Zeitpunkt zwei Brandherde hell lodernd brannten. Die Abb. 6 zeigt dieses Feuer.

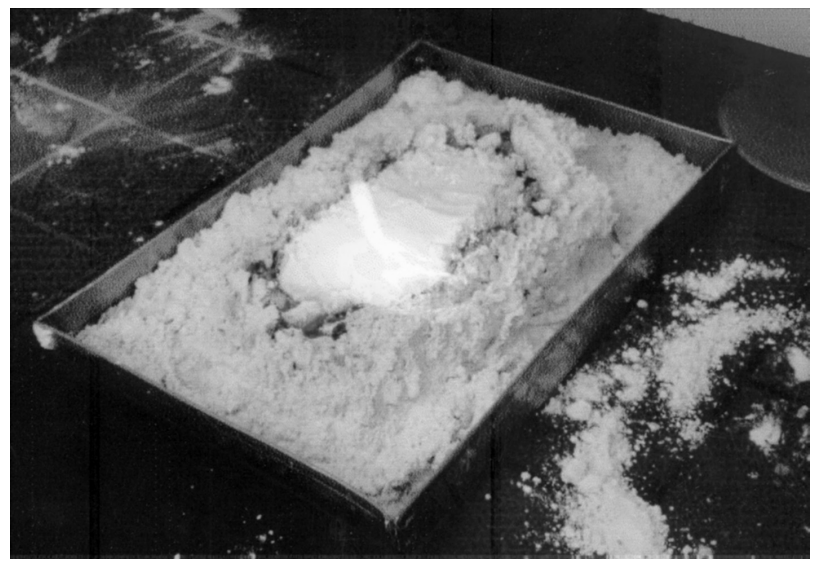

Abb. 6: Das Ergebnis des erfolgreichen Großversuches: Der in der mittelalterlichen Literatur überlieferte chemische Anzünder auf $\mathrm{CaO}$-Basis hat primär Schwefel, sekundär Petroleum entzündet, hat somit erfolgreich gearbeitet - allerdings erst nach einer tiefgreifenden Modifikation des ursprünglichen Rezeptes und nur mit einer Wirkrate von unter $50 \%$. (farbige Abbildung im Internet: http://www.edition-sigma.de/TG/H1_07/AbbNibler.PDF).

Ungefähr 20 Minuten nach Reaktionsbeginn war etwa die Hälfte des Schwefels verbrannt, das noch gut brennende Feuer wurde aber aus Zeitgründen gelöscht und der Versuch beendet. 
Mit der großen Masse von $\approx 2,5 \mathrm{~kg}$ Kalk wurden nun weitere Versuche gemacht, um das positive Ergebnis des ersten Versuches zu verifizieren. Dabei zeigte sich, dass das Verhältnis Kalk zu Schwefel ganz unkritisch ist und keineswegs ,an der wag“ (so FWB 229) eingestellt werden muss, und dass auch die Wassermenge weniger kritisch ist als bei geringen Reaktionsmassen. Damit konnte ein Primärfeuer häufig entzündet werden, ein Sekundärfeuer aber deutlich weniger oft, nämlich nur in knapp 50\% der Fälle. Zu erwähnen ist allerdings, dass der Zeitablauf von Versuch zu Versuch sehr unterschiedlich sein konnte. Der oben detailliert geschilderte Versuchsablauf ist als „,schnell“ zu bezeichnen, „langsame“ Versuchsabläufe, bei denen die erste Entzündung des Schwefels erst nach 15 Minuten auftrat, die Entzündung des Petroleums sogar erst nach 20 Minuten, waren nicht ungewöhnlich.

\section{Fazit}

Die diversen Rezepte für automatische Kriegsfeuer enthalten neben offensichtlich wenig sinnvollen Angaben auch einen realen Kern, d.h. die exotherme Reaktion des Kalklöschens kann tatsächlich zum Entzünden eines Feuers benutzt werden. Der sich bei $260^{\circ} \mathrm{C}$ entzündende Schwefel brennt allerdings mit so kleiner Flamme, dass von der in den Rezepten beschriebenen starken Wirkung keine Rede sein kann: Die in den Rezepten angegebenen Mengen in der Größenordnung von Unzen sind zu klein, die erforderliche Dosierung des Wassers zu kritisch, so dass den ,automatischen“ Feuern der Typen 1 und 2 mit geringen Massen der praktische Nutzen im Kriegseinsatz eindeutig abgesprochen werden muss.

Wenn die Massen der reagierenden Materialien allerdings deutlich auf ein oder mehr Kilogramm erhöht werden, was nur im Fall zweier Rezepte des 15. Jahrhunderts (FWB) im „Pfundbereich“ einigermaßen zu belegen ist, dann ist es zumindest unter Laborbedingungen möglich, mit einem Brandsatz, der Material (z.B. Petroleum) für ein Sekundärfeuer enthält, über dieses Sekundärfeuer ein Schadfeuer zu entzünden. Da das Wasser aber auch hier zumindest grob dosiert und zudem sehr schnell zugeführt werden muss, kann der „Sabotage-Einsatz mit Brandauslösung durch Regen" ${ }^{28}$ nicht erreicht werden.

Wenn überhaupt, dann ist der chemische Anzünder nur als eine Art „Feuerzeug" brauchbar - das aber ist in der damaligen Zeit als Alternative zu „Feuerstein, Stahl und Zunder" sicher nicht zu unterschätzen! Das unvermeidlich skeptisch-abschätzige Gesamturteil, zu dem man abschließend kommen muss, passt dazu, dass zwar relativ viele Rezepte überliefert sind, aber keine konkreten Erfolgsmeldungen oder wenigstens Nachrichten über versuchte Einsätze bekannt sind, und es bedeutet, dass die meisten, wenn nicht alle, Rezepte ausgesprochenen Unsinn enthalten, weil sie in der überlieferten Fassung nicht funktionieren. 
Dieses abschließende negative Urteil deckt sich mit dem Ergebnis von vergleichbaren Experimenten, die 1965 von Dittrich gemacht wurden, um das „Griechische Feuer“ zu untersuchen. Dittrich stellte dazu lapidar fest: „Eine Mischung bestehend aus Schwefel, Kolophonium, Petroleum, Teer und Kochsalz, der man ungelöschten Kalk zugesetzt hat, damit sie sich beim Löschen mit Wasser entzündet, brennt nicht ${ }^{\text {“ }}{ }^{29}$ Weitere experimentelle Arbeiten zum Thema sind dem Verfasser nicht bekannt.

Erwähnenswert erscheint aber, dass auch Partington dem $\pi v \rho \alpha v \tau o \mu \alpha \tau o v$, dem ,automatischen“ Feuer, sehr skeptisch gegenübersteht. An einer Stelle sagt er beispielsweise ,the mixture described would not inflame when ,the sun rises '... but it might inflame sooner or later if exposed to ... dew or light rain. Such an uncertain weapon ... would not be viewed with favour by military commanders". ${ }^{30}$ Dass ungelöschter Kalk aber durchaus eine potentielle Brandursache sein kann, wird andererseits durch die amtlichen bayerischen Lagervorschriften für ungelöschten Kalk in der aktuellen Verordnung über die Verhütung von Bränden bestätigt. ${ }^{31}$ Zusammenfassend lässt sich also feststellen, dass mit ungelöschtem Kalk keine verlässlich funktionierenden Anzünder hergestellt werden können und dass die überlieferten Rezepte für ,,automatische" Kriegsfeuer unbrauchbar sind, obwohl bei der Lagerung von ungelöschtem Kalk ein gewisses Brandrisiko besteht.

Anschrift des Verfassers: Prof. Dr. Ferdinand Nibler, Robert-Koch-Str. 13d, 85521 Ottobrunn. E-Mail: Dr.F.Nibler@t-online.de.

29 Bericht aus dem Chemischen Laboratorium des Bundesministeriums für Landesverteidigung Wien XI, Leitung Dipl.-Ing. Oberstleutnant Dittrich, vom 11.3.1965: Betr.: Überprüfung von alten Rezepten für „Griechisches Feuer“. Zitiert nach Kurt Frischler, Wunderwaffen - 5000 Jahre Jagd nach der absoluten Macht, Wien u. München 1965, S. $164 f$.

30 James R. Partington, A History of Greek Fire and Gunpowder, Cambridge 1960, Reprint Baltimore u. London 1999, S. 9.

31 Verordnung über die Verhütung von Bränden (VVB) vom 29. April 1981 (BayRS 215-21-I), zuletzt geändert durch Verordnung vom 7. November 2004 (GVBl S. 450). 
